\title{
Stimulating the phagocytes: progress and problems
}

\begin{abstract}
Nature has provided, in the white corpuscles as you call them - in the phagocytes as we call them - a natural means of devouring and destroying all disease germs. There is at bottom only one genuinely scientific treatment for all diseases, and that is to stimulate the phagocytes. Stimulate the phagocytes. Drugs are a delusion.
\end{abstract}

[The Doctor's Dilemma, George Bernard Shaw,

In the early days of the description of phagocytes and the first wave of (unsuccessful) antituberculosis therapy, Shaw recognised and publicised that the phagocytes were exquisitely interested in ingesting and killing bacteria, that that act was dependent on opsonisation and that the activity of phagocytes was stimulable. From the modern vantage point of effective antibiotics we do not think drugs a delusion. However, the necessary role of phagocyte stimulation in the prevention and clearance of certain intracellular infections underscores the fact that for certain infections, antibiotics remain adjuncts to effective phagocytic killing.

For over three decades, rare occurrences of familial disseminated non-tuberculous mycobacterial (DNTM) infections have been identified in the absence of HIV. In the 15 years since the rise of HIV, DNTM disease has been recognised as a characteristically immunodeficient presentation of this infection [1]. With the brighter light cast on immunodeficiency by HIV, these patients have taken on new importance. Recently, the underlying defects in some of these cohorts have been identified at the molecular and immunological levels. Mutations in the ligand binding $[2,3]$ and signal transducing [4] chains of the interferon- $\gamma$ receptor and mutations in the $\beta 1$ chain of the interleukin-12 (IL-12) receptor $[5,6]$ have been identified in patients with DNTM infections or salmonellosis. Abnormalities in IL-12 regulation have also been identified in patients with DNTM disease [7]. All these patients have a relatively narrow spectrum of infections, so far limited to the mycobacteria and salmonellae.

From an immunological standpoint, the general pathways (although not the mechanisms) involved in the host response to intracellular pathogens are fairly well described $[1,8]$. In the case of mycobacteria, organisms infect the macrophage, stimulating the production of IL-12. IL-12 acts on lymphocytes to stimulate the production of IL-2, leading to the expansion of responding clones and the production of interferon- $\gamma$. Interferon- $\gamma$ acts through its cognate receptor to elicit a variety of responses, among which are the upregulation of tumour necrosis factor- $\alpha$ (an activity greatly enhanced by simultaneous exposure to endotoxin), the upregulation of IL-12 and killing of intracellular parasites such as mycobacteria and salmonellae. Therefore, interferon- $\gamma$ is critically required for its own upregulation [9]. There are several counter-regulatory pathways (e.g., IL-10 and transforming growth factor- $\beta$ ) involved in controlling interferon- $\gamma$ production, but none of these has yet been identified as causing disease in man.

It appears that the critical role for this complex circuit, at least where intracellular infections are concerned, is to increase interferon- $\gamma$ concentrations. Patients with complete absence of the interferon- $\gamma$ receptor fail to form granulomata and develop DNTM infections that are frequently fatal [3]. In contrast, patients with defects in the IL-12 receptor retain a low level of interferon- $\gamma$ production, which appears to account for the ability of these patients to form morphologically normal granulomata and to exhibit a much higher survival rate $[5,6]$. Patients with defects in the IL-12 receptor, or in IL-12 production, are successfully treated for their DNTM infections with interferon- $\gamma$, along with antimycobacterial agents, and have not been reported to get infections other than those caused by mycobacteria and salmonellae $[5,6,10]$.

Why is interferon- $\gamma$ so critically important in the control of these intracellular infections? Of the $>200$ genes known to be regulated by interferon- $\gamma$ [11], one would guess that only a small but critical number were silenced completely by a specific lesion in the interferon- $\gamma$ receptor. Therefore, the receptor must have a few non-redundant properties that are required for the control of mycobacteria and salmonellae. The mechanisms that mediate the activity of interferon- $\gamma$ against intracellular infections are still not clearly defined. Candidate activities include increased antigen processing and presentation, lowering of intravacuolar $\mathrm{pH}$, increased production of superoxide and its downstream metabolites, decreased prostaglandin pro- 
duction and increased intracellular antibiotic concentration $[8,12]$. It is likely that the role of interferon- $\gamma$ in preventing DNTM infection in normal hosts is somewhat different from the activity it exerts in the treatment of established infections. In the treatment of leprosy [13] and leishmaniasis $[14,15]$ with interferon- $\gamma$, reduction in microbial burden is accomplished only when it is given in conjunction with antibiotics; no reduction in infectious burden is noted when interferon- $\gamma$ is given alone. Further determination of the exact mechanism of interferon- $\gamma$ action is critical if therapy is to be targeted successfully to the most sensitive point in the intracellular armamentarium.

The potential applications of interferon- $\gamma$ in man are still largely undeveloped. Presently, the only licensed use in the USA is in the prophylaxis of infections in chronic granulomatous disease, a phagocytic disorder not associated with any kind of interferon- $\gamma$ deficiency [16]. Trials showing human clinical therapeutic benefit have been completed in leprosy, leishmaniasis and DNTM disease [12]. Studies in the treatment of pulmonary non-tuberculous mycobacterial infections and pulmonary tuberculosis with either systemic or aerosolised interferon- $\gamma$ are under way and show early promise $[8,17]$. Trials in renal carcinoma, post-trauma patients, atopic dermatitis, rheumatoid arthritis, scleroderma and neonates have not shown particular benefit. Areas predicted from mouse models, but as yet unexplored in human therapeutic trials, include fungal infections, persistent viral infections, and persistent bacterial infections.

There are several impediments to broadening the therapeutic horizons of interferon- $\gamma$. Like most of the marketed cytokines, interferon- $\gamma$ is quite expensive and clinical experience is therefore limited. Because good drugs exist for many of the diseases, the impetus to explore novel and expensive agents is low. However, the real benefit of interferon- $\gamma$ in normal hosts may be in shortening the duration of therapy, thereby reducing exposure to toxic drugs and increasing compliance. For those with refractory infections and intact or partially intact receptors, interferon- $\gamma$ may be the only successful adjuvant therapy available. There may be other infections that are highly virulent in interferon- $\gamma$ receptor-deficient individuals, which are unrecognised at present because they occur in locations or populations that have not yet been studied. If the mouse models are predictive, increased viral susceptibility, as well as impaired handling of common bacterial infections such as those caused by staphylococci, might be expected.

The identification of individuals with mutations in the interferon- $\gamma$ receptor and those with mutations in the interferon- $\gamma$ inducing IL-12 receptor has been critical in confirming a role for interferon- $\gamma$ in human disease. Shaw was prescient in his recognition of the critical role of phagocyte stimulation in the control of disease. However, until our knowledge of the mechanism of action is a bit deeper and our development of therapy more successful, we should stick to our current delusions about the conventional role of drugs and keep the phagocyte-stimulating cytokines as adjuvants to explore in controlled trials and cases of extreme need.

S. M. Holland Laboratory of Host Defenses, National Institute of Allergy and Infectious Diseases, National Institutes of Health, Bethesda,

MD 20892-1886, USA e-mail:smh@nih.gov

\section{References}

1. Holland SM. Host defense against nontuberculous mycobacterial infections. Semin Respir Infect 1996; 11: 217-230.

2. Newport MJ, Huxley CM, Huston $\mathrm{S}$ et al. A mutation in the interferon- $\gamma$-receptor gene and susceptibility to mycobacterial infection. $N$ Engl J Med 1996; 335: 1941-1949.

3. Jouanguy E, Altare F, Lamhamedi S et al. Interferon- $\gamma$-receptor deficiency in an infant with fatal bacille Calmette-Guérin infection. $N$ Engl J Med 1996; 335: 1956-1961.

4. Dorman SE, Holland SM. Mutation in the signal-transducing chain of the interferon- $\gamma$ receptor and susceptibility to mycobacterial infection. $J$ Clin Invest 1998; 101: 2364-2369.

5. de Jong R, Altare F, Haagen I-A et al. Severe mycobacterial and salmonella infections in interleukin-12 receptor-deficient patients. Science 1998; 280: 1435-1438.

6. Altare F, Durandy A, Lammas D et al. Impairment of mycobacterial immunity in human interleukin-12 receptor deficiency. Science 1998; 280: 1432-1435.

7. Frucht DM, Holland SM. Defective monocyte costimulation for IFN- $\gamma$ production in familial disseminated Mycobacterium avium complex infection. Abnormal IL-12 regulation. $J$ Immunol 1996; 157: 411-416.

8. Holland SM. Therapy of mycobacterial infections. Res Immunol 1996; 147: 572-581.

9. Holland SM, Dorman SE, Kwon A et al. Abnormal regulation of interferon- $\gamma$ interleukin 12, and tumor necrosis factor- $\alpha$, in human interferon- $\gamma$ receptor 1 deficiency. J Infect Dis 1998; 178: $1095-1104$

10. Holland SM, Eisenstein EM, Kuhns DB et al. Treatment of refractory disseminated nontuberculous mycobacterial infection with interferon gamma. A preliminary report. $N$ Engl J Med 1994; 330: $1348-1355$.

11. Boehm U, Klamp T, Groot M, Howard JC. Cellular responses to interferon- $\gamma$. Annu Rev Immunol 1997; 15: 749-795.

12. Gallin JI, Farber JM, Holland SM, Nutman TB. Interferon- $\gamma$ in the management of infectious diseases. Ann Intern Med 1995; 123: $216-224$

13. Nathan CF, Kaplan G, Levis WR et al. Local and systemic effects of intradermal recombinant interferon- $\gamma$ in patients with lepromatous leprosy. $N$ Engl J Med 1986; 315: 6-15.

14. Badaro R, Falcoff E, Badaro FS et al. Treatment of visceral leishmaniasis with pentavalent antimony and interferon gamma. $N$ Engl J Med 1990; 322: 16-21.

15. Sundar S, Murray HW. Effect of treatment with interferon- $\gamma$ alone in visceral leishmaniasis. $J$ Infect Dis 1995; 172: $1627-1629$.

16. The International Chronic Granulomatous Disease Cooperative Study Group. A controlled trial of interferon gamma to prevent infection in chronic granulomatous disease. $N$ Engl $J$ Med 1991; 324: 509-516.

17. Condos R, Rom WN, Schluger NW. Treatment of multidrug resistant pulmonary tuberculosis with interferon gamma via aerosol. Lancet 1997; 349: 1513-1515. 